\title{
The cellular pathways of liver fibrosis in non-alcoholic steatohepatitis
}

\author{
Nahum Méndez-Sánchez ${ }^{1,2}$, Alejandro Valencia-Rodríguez ${ }^{1}$, Carlos Coronel-Castillo ${ }^{1}$, \\ Alfonso Vera-Barajas ${ }^{1}$, Jocelyn Contreras-Carmona ${ }^{1}$, Guadalupe Ponciano-Rodríguez ${ }^{3}$, \\ Daniel Zamora-Valdés ${ }^{1}$
}

${ }^{1}$ Liver Research Unit, Medica Sur Clinic \& Foundation, Mexico City, Mexico; ${ }^{2}$ Faculty of Medicine. National Autonomous University of Mexico, Mexico City, Mexico; ${ }^{3}$ Public Health Department, Faculty of Medicine, National Autonomous University of Mexico, Mexico City, Mexico Correspondence to: Prof. Nahum Méndez-Sánchez, MD, MSc, PhD, FACG, AGAF. Liver Research Unit, Medica Sur Clinic and Foundation, National Autonomous University of Mexico, Puente de Piedra 150, Col. Toriello Guerra, ZP. 14050, Mexico City, Mexico.

Email: nmendez@medicasur.org.mx; nah@unam.mx.

\begin{abstract}
Non-alcoholic steatohepatitis (NASH) is considered the advanced stage of non-alcoholic fatty liver disease (NAFLD). It is characterized by liver steatosis, inflammation and different degrees of fibrosis. Although the exact mechanisms by which fatty liver progresses to NASH are still not well understood, innate and adaptive immune responses seem to be essential key regulators in the establishment, progression, and chronicity of these disease. Diet-induced lipid overload of parenchymal and non-parenchymal liver cells is considered the first step for the development of fatty liver with the consequent organelle dysfunction, cellular stress and liver injury. These will generate the production of pro-inflammatory cytokines, chemokines and damage-associated molecular patterns (DAMPs) that will upregulate the activation of Kupffer cells (KCs) and monocyte-derived macrophages (MMs) favoring the polarization of the tolerogenic environment of the liver to an immunogenic phenotype with the resulting transdifferentiation of hepatic stellate cells (HSCs) into myofibroblasts developing fibrosis. In the long run, dendritic cells (DCs) will activate CD4+ T cells polarizing into the pro-inflammatory lymphocytes Th1 and Th17 worsening the liver damage and inflammation. Therefore, the objective of this review is to discuss in a systematic way the mechanisms known so far of the immune and non-proper immune liver cells in the development and progression of NASH.
\end{abstract}

Keywords: Immune system; liver cells; liver fibrosis; non-alcoholic steatohepatitis (NASH)

Submitted Feb 08, 2019. Accepted for publication Feb 29, 2020.

doi: $10.21037 /$ atm.2020.02.184

View this article at: http://dx.doi.org/10.21037/atm.2020.02.184

\section{Introduction}

The liver is one of the most regenerative tissues in the body with the capacity to regenerate itself even after partial hepatectomy. Despite this, there is critical difference between the response to transient or chronic liver damage (1). Usually, after acute injury the liver will be able to return to its original architecture by proliferation and remodeling of the remaining cells trough the interaction of the distinct innate immune liver cells such as Kupffer cells (KCs), dendritic cells (DCs), neutrophils and innate-like lymphocytes (ILCs) with parenchymal cells like hepatocytes and liver sinusoidal endothelial cells (LSECs) without completely losing the characteristic tolerogenic capacity of this organ (2-4). The balance between the immune response, the grade of apoptosis, the grade of cells mitosis, and the grade of liver injury is important for the recovery of hepatic tissue $(4,5)$.

In contrast to acute injury, chronic liver injury overcomes the regenerative capacity of the liver resulting in fibrosis and its further complications. Fibrosis is an adaptive mechanism with the primary objective of repair the damaged tissue, however, after prolonged injury it can progress to parenchymal scarring, cellular dysfunction and finally to organ failure by the activation of hepatic stellate cells 
(HSCs), characteristic of liver cirrhosis (6). In this context, non-alcoholic steatohepatitis (NASH) has emerged in the last decade as an important cause of worldwide cirrhosis with scary projections for the near future $(7,8)$. Although the exact mechanisms by which non-alcoholic fatty liver disease (NAFLD) progresses to NASH are still not completely understood, it is known that liver cells of the innate immune response play an important role in the shift of liver's tolerogenic environment to an immunogenic phenotype through the recognition of a myriad of danger signs known as damage-associated molecular patterns (DAMPs) released from damaged parenchymal and non-parenchymal liver cells, and also the recognition of bacterial products from enterohepatic circulation known as pathogen-associated molecular patterns (PAMPs) via pattern-recognition receptors (PRRs) from immune cells. Moreover, adaptive immune response also plays an important role in NASH progression. Recent studies in human and animal models have found lymphocytic infiltrates in advanced NASH stages associated with a worse degree of steatohepatitis and fibrosis (9-12) making the comprehension of those cells an important issue of study. With this in mind, it is the objective of this review to discuss in a systematic way the mechanisms known so far of the immune and non-proper immune liver cells in the development and progression of NASH.

\section{Overview of the development of NAFLD}

Currently, many metabolic diseases are the direct consequence of an overnutrition state combined with the lack of physical exercise (13). In the case of NAFLD, we can see that a high-calorie diet leads to insulin resistance (IR) decreasing its antilipolytic effect on adipose tissue (AT), generating the breakdown of triglycerides (TGs) though the hydrolysis of 3 enzymes; adipose triglyceride lipase (ATGL), hormone-sensitive lipase (HSL), and monoglyceride lipase (MGL) with the consequent formation of free fatty acids (FFAs) and glycerol (14). Also, AT dysfunction will follow a lower production of adiponectin and a greater release of adipokines such as leptin, conditioning a low-grade proinflammatory state (15).

An increase in circulating FFAs in the systemic circulation enhances a higher uptake of FFAs by the liver leading to an accumulation of lipids in liver cells (16). Interestingly, not all dietary lipids have cytotoxic effects, monounsaturated fatty acids (MUFAs) and polyunsaturated fatty acids (PUFAs) like docosahexaenoic acid (an omega-3 fatty acid) have shown protective actions by binding directly to the peroxisome proliferator activated receptor- $\alpha$ $(\mathrm{PPAR}-\alpha)$ in association with the retinoid $\mathrm{X}$ receptor (RXR) creating and heterodimeric complex (PPAR- $\alpha /$ RXR) involved in fatty acid oxidation and regulation of TGs catabolism (17) while saturated fatty acids (SFAs) like palmitic and stearic acid promote inflammation by indirect Toll-like receptors (TLRs) signaling favoring NAFLD development (18).

Besides, IR and hyperglycemia favors hepatic de novo lipogenesis (DNL) via sterol regulatory element binding protein 1c (SREBP1c) setting up the creation of lipid droplets in the liver parenchyma (19). Fructose-derived precursors also act as nutritional regulators of hepatic DNL via SREBP1c and carbohydrate response element binding protein (ChREBP) signaling (20).

The excessive accumulation of toxic lipids in the liver (a process commonly known as lipotoxicity) is associated with organelles dysfunction, mainly endoplasmic reticulum (ER) and mitochondria (Figure 1). Lipotoxicity causes ER stress by deregulating its reaction to misfolded proteins creating an aberrant response of the 3 intracellular pathways of the ER (21). In this context the overactivation of RNAdependent protein kinase-like ER eukaryotic initiation factor-2 $\alpha$ kinase (PERK) and activating transcription factor 6 (ATF6) increases the secretion of proinflammatory cytokines via nuclear factor- $\kappa \beta(\mathrm{NF}-\kappa \beta)$ pathway. The other intracellular pathway of the ER known as inositol-requiring enzyme 1 (IRE1) is also associated with the release of proinflammatory cytokines though TNF $\alpha$ receptorassociated factor 2 (TRAF2) binding via kinase 1 (19).

In the case of mitochondrial stress, lipid overload increases acetyl-CoA synthesis disturbing the tricarboxylic acid (TCA) cycle function from mitochondrial respiration enhancing reactive oxygen species (ROS) formation (22). Moreover, the alteration on $\beta$-oxidation process will end up with the formation of toxic lipid intermediates like ceramides (23).

Once NAFLD is established, the combination of lipotoxicity and the low-grade inflammation will determine an immune response by liver cells with two main objectives: (I) activate innate immune cells to "control the damage"; and (II) repair the damaged tissue. Paradoxically, in some cases this will end up in the development of NASH. For this reason, the conceptualization of each one of these immune cells must be seen as a dynamic process and not as a series of steps to follow, however, for a better understanding, the main mechanisms of the cells involved in this process will be 


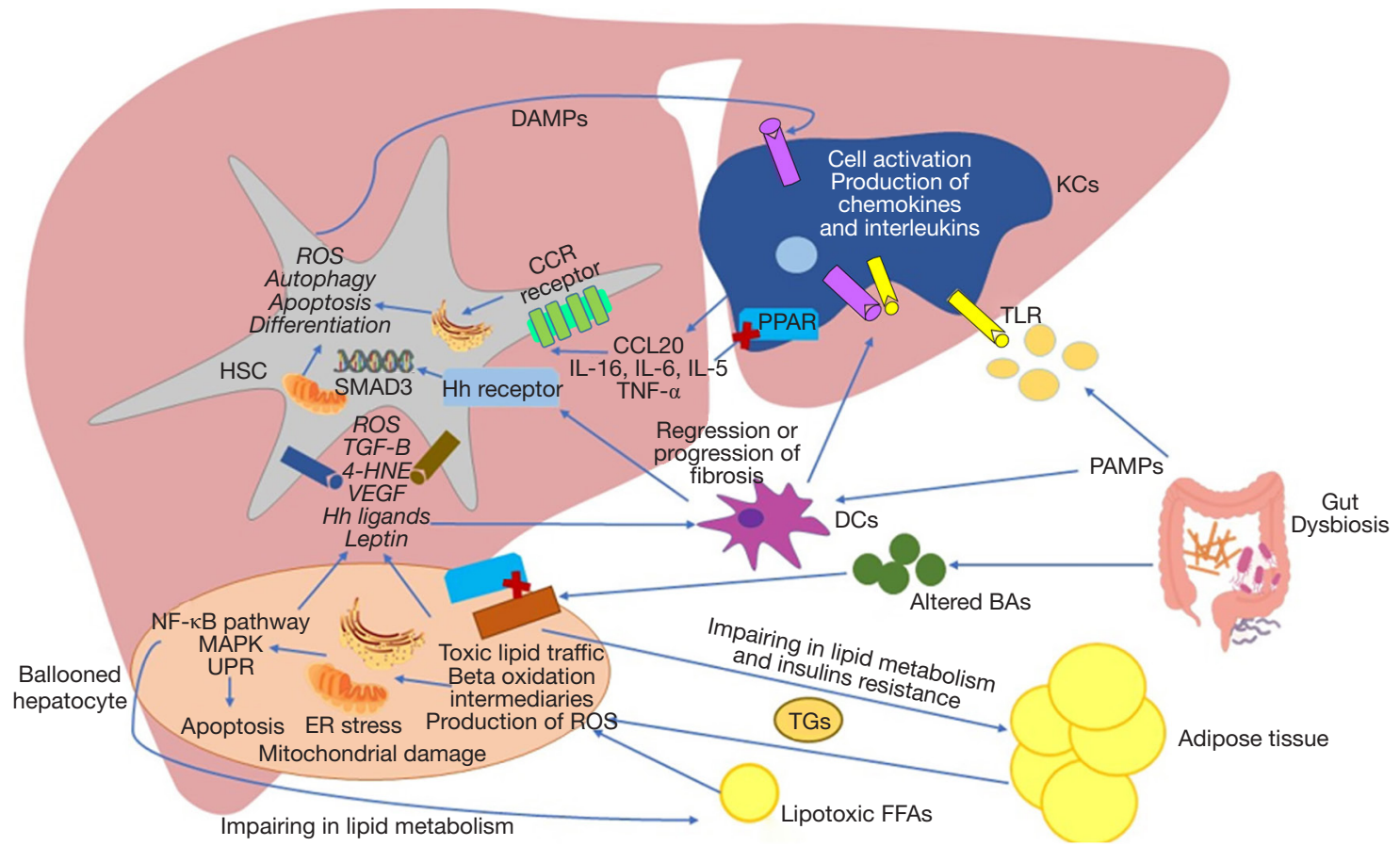

Figure 1 Crosstalk between liver cells and their response to different stimuli. Hepatocytes, Kupffer cells (KCs), hepatic stellate cells (HSCs) and dendritic cells (DCs) are the most important liver cells within the development of non-alcoholic steatohepatitis (NASH). Diet-induced lipid overload will generate lipotoxicity and glucotoxicity with the consequent endoplasmic reticulum (ER) and mitochondrial stress inducing the formation of reactive oxygen species (ROS) and a deregulated unfolded protein response (UPR) developing apoptosis and liver injury. Damage-associated molecular pattern (DAMPs) will activate myeloid-derived cells promoting inflammation and the transdifferentiation of HSCs to myofibroblasts developing fibrosis.

described in a systematic way in the following paragraphs.

\section{Hepatocyte}

The hepatocytes are the main liver parenchyma cells representing around $85 \%$ of the liver mass (24). They are considered the main functional cells of the liver as they have several functions just as protein synthesis and storage, carbohydrate metabolism, bile formation, drug and toxic catabolism, among others. Within NAFLD development, these cells suffer a characteristic variation in its structure developing lobular inflammation and balloon degeneration associated with different degrees of scarring or fibrosis (25) due to the release of proinflammatory cytokines like tumor necrosis factor- $\alpha$ (TNF- $\alpha$ ), interleukin (IL-6) and (IL$1 \beta$ ) derived from organelles dysfunction. Also, ER stress promotes proteotoxicity and proapoptotic signals through a rapid decay of selected microRNAs that would normally suppress apoptosis (26-28). The C/EBP homologous protein $(\mathrm{CHOP})$ is one of the most important proapoptotic signals activated by PERK and ATF6 pathways (29). Besides, the phosphorylation of IRE1 is associated with the activation of the c-Jun $\mathrm{N}$-terminal kinase (JNK) (28), the $\mathrm{Bcl}-2$-associated $\mathrm{X}$ protein (BAX) and $\mathrm{Bcl}-2$ homologous antagonist killer (BAK) pathways related with apoptosis (30). On the other hand, ROS-derived from mitochondrial dysfunction result in decreased levels of adenosine triphosphate (ATP) and in the depletion and inhibition of antioxidant molecules such as thioredoxin and glutathione $(31,32)$. Also, the oxidization of SFAs leads to the production of aldehyde byproducts like 4-hydroxy-2nonenal (HNE) and malondialdehyde (MDA) perpetuating protein oxidation and lipid peroxidation (33). These mechanisms may eventually result in a deleterious cycle of mitochondrial damage and mitochondria-originating oxidative stress (34). Oxidative stress is essential for NASH progression, as it has been reported to activate NF-k $\beta$ pathway inducing the production of pro-inflammatory cytokines enhancing apoptosis and necrosis in hepatocytes (35-37). Damaged mitochondria and the subsequent 
necrosis of hepatocytes release a set of mitochondriaderived danger molecules known as DAMPs, like the high mobility group box protein (HMGB1), alarmins, nucleic acids, histones, ATP and uric acid which can be recognized by the PRRs of the myeloid cells and induce the activation of the innate immune response. Also, several experimental models with mice have shown that mitochondrial DNA (mtDNA) interacts with TLR-9 on KCs and HSCs stimulating the innate immune and fibrogenic responses (38-40). Finally, another way that hepatocytes have to stimulate the activation of myeloid cells, is the release of extracellular vesicles (EVs). These molecules are important mediators in the crosstalk of cell to cell communication initiating or suppressing signaling pathways in the recipient cell through the transfer of certain types of biomolecules. Recently one of this type of EVs known as exosomes has been discovered to transport the chemokine CXCL10 and ceramides to KCs with the capacity of recruit neutrophils via IL-8 (41) and activate macrophages via sphingosine-1-phosphate (42). Also, palmitate was seen to induce the liberation of EVs by the hepatocytes containing TNF-related apoptosis-inducing ligand (TRAIL), an important proapoptotic protein with the ability to activate macrophages (43), both representing important mechanisms in disease progression.

\section{$K C$}

KCs are the resident liver macrophages which are part of the reticuloendothelial system (RES) of this organ. They play a critical role in the mononuclear phagocytic system essential to both the hepatic and systemic response to pathogens (44). A dysregulation in the control of inflammatory responses in KCs and other macrophages can contribute to chronic liver inflammation (45). Interestingly, KCs and bone marrowderived macrophages (BMMs) have different roles in NAFLD pathogenesis depending on the current state of the liver injury. In acute phase, KCs and BMMs are polarized into their activated phenotype (M1) though the recognition of DAMPs or PAMPs by specialized PRRs just as TLRs and NOD-like receptors (NLRs) (46-49). HMGB1, one of the most important DAMPs released by damaged cells activates TLR4 and promoted the binding with its ligand myeloid differentiation primary response 88 (MYD88) resulting in a multiple activation of $\mathrm{JNK}$, inhibitor of nuclear factor kappa-B kinase 2 (IKK2) and mitogenactivated protein kinase (MAPK) p38 with the consequent expression of the NF- $\kappa \beta$ pathway and the activation of the activator protein 1 (AP-1) leading to the release of TNF- $\alpha$, interferon (IFN- $\gamma$ ), prostaglandin-2 (PGE2), chemokine C-C motif ligand (CCL), IL- $1 \alpha$, IL-1 $\beta$, IL-6, ROS, and nitric oxide (NO) (50). SFAs can induced by themselves the activation of liver macrophages via TLR4 and TLR2 (51). Also, other types of danger signals can promote the activation of intracellular multiproteic oligomers known as inflammasomes by the NLRs binding and the secretion of proinflammatory cytokines (52). Similarly, fructose is another diet-component with the capacity of activate the inflammasome NLRP3 in macrophages via thioredoxininteracting protein (TXNIP) (53) with the expression of CCL2, CXCL2, IL-6, and TNF- $\alpha$ (54). All those cytokines and chemokines can recruit non-resident cells to the liver like neutrophils, natural killer T cells (NKT), $\mathrm{CD}^{+}$and $\mathrm{CD}^{+} \mathrm{T}$ cells (55). An important number of studies have found that KCs and BMMs recruited to the liver in acute liver injury showed an increased expression of TNF- $\alpha$, IL$1 \beta$ and CCR2 promoting NASH progression (56-58).

However, as we mentioned, KCs and BMMs show a different phenotype in chronic phase since most of these cells change to their anti-inflammatory phenotype (M2) by multiple pathways (59). One of them is through an increase in the conversion of oxidized low-density lipoproteins (LDL) to oxysterols by the cytochrome P450 oxidase and with the further binding of oxysterols with liver X receptor (LXR) inhibiting the NF- $\kappa \beta$ pathway (60). Additionally, in chronic stages adiponectin may increase the levels of adenosine monophosphate (AMP) leading to the activation of the AMP kinase (AMPK), inhibiting acetyl Co-A carboxylase, increasing the FFAs oxidation, and inhibiting SREBP-1C (61). Finally, the RAR-related orphan receptor- $\alpha(\mathrm{ROR} \alpha)$ pathway via factor 4 similar to Kruppel (KLF4) induced by PUFAs through an increased expression of PPAR- $Y$ are another important anti-inflammatory mechanism of liver macrophages (19). In a recent study, BMMs depletion in mice with advanced NASH conditioned a significant increase in the activation of HSCs with the consequent production of collagen fibers and therefore a worsen liver histology (62). These results demonstrate a probable polarization of macrophages to their antiinflammatory phenotype (M2) in stages of liver recovery by inducing the expression of anti-inflammatory cytokines like IL-4, IL-5 and IL-10 (50). In spite of this, more studies are required to know the specific mechanisms by which hepatic macrophages change their phenotype in advanced stages 
Table 1 Main pathways involved in HSCs activation

\begin{tabular}{ll}
\hline Cells & Molecules \\
\hline Hepatocytes & CTGF \\
KCs & PDGF $\beta$ \\
T Cells & TGF $\beta$ \\
DCs & VEGF \\
Neutrophils & Interleukins and TNF- $\alpha$ \\
Adipocytes & Leptin and adiponectin \\
& PAMPs (TLR) \\
& DAMPs \\
& ROS \\
\hline
\end{tabular}

KCs, Kupffer cells; DCs, dendritic cells; CTGF, connective tissue growth factor; PDGF $\beta$, platelet-derived growth factor $\beta$; TGF $\beta$, transforming growth factor $\beta$; VEGF, vascular endothelial growth factor; PAMPs, pathogen-associated molecular patterns; TLR, Toll-like receptor; DAMPs, damage-associated molecular patterns; ROS, reactive oxygen species.

of NASH.

\section{HSC}

HSCs are a type of pericytes found in the perisinusoidal space of the liver with the capacity of storage a great amount of lipid droplets containing vitamin A as retinol esters. Under physiological conditions, HSCs are under a quiescent phenotype (qHSCs) with a highly production of IFNY via expression of TLR3 (63), however, in chronic liver disease HSCs suffer from a transdifferentiation into myofibroblasts losing the ability to produce IFNY but becoming the most important cellular source of matrix protein-secretion (considered de major driver of liver fibrosis) (49).

Several pathways have been dilucidated in the activation of HSCs (Table 1), from these the most significant are described below (49).

* Transforming growth factor- $\beta$ (TGF- $\beta$ ) is considered the most important fibrinogenic cytokine by promoting the transcription of type I and type III collagen as well as the mitogen-activated protein kinase (MAPK) and JNK signaling pathways favoring the HSCs activation.

* The platelet derived growth factor (PDGF) is an important chemoattractant for HSCs proliferation and migration.
* Vascular endothelial growth factor (VEGF) is produced mainly by LSECs and HSCs promoting fibrogenesis, but also required for hepatic tissue repair and fibrosis resolution.

* Connective tissue growth factor (CTGF) is a potent fibrogenic cytokine highly express in liver fibrosis contributing to extracellular matrix production as well as proliferation, migration, adhesion and survival of liver cells.

In addition to these pathways, patients with NASH reveal high concentration of leptin inversely proportional to the levels of adiponectin favoring the profibrotic effect (64). In this context, a recent meta-analysis showed that circulating leptin concentrations were proportional with NAFLD severity (65). Leptin can be secreted by AT, $\mathrm{KCs}$ and other non-parenchymal cells in the liver as an important signal traductor for HSCs, thus, it is considered a potent mitogenic agent. In both in vivo and in vitro studies leptin promotes HSCs proliferation and inhibits cells apoptosis with effects nearly as potent as PDGF (66). At the same time, the role of interleukins secreted by KCs are also important for HSCs differentiation. Liu et al. found in a mice-model with liver fibrosis an important upregulation of KCs activity as well as an increased expression of TNF- $\alpha, \alpha$-smooth muscle actin ( $\alpha$-SMA) and collagen type I-positive cells that interestingly do not underwent apoptosis (67). Alternatively, the chemokine CCL20 which is highly up-regulated in NAFLD-associated fibrosis seems to be released by HSCs in response to lipid loading (68). This means that HSCs are also capable to induce fibrosis by themselves. Recent findings have shown that free cholesterol (FC) may induce HSCs activation by direct signaling of TLR4 (69). Therefore, this could be a key mechanism in the fibrotic progression of NAFLD in response to the increased caloric intake in obesity (70). Another chemokine that is expressed in NASH, particularly in early stages, is CCL5. In a study with mice fed with a choline-deficient diet for three weeks, it results in a developing of NASH with an increase expression of Ccl5 secreted by HSCs (71).

Finally, the role of the hepatic endocannabinoid (EC) and the apelin systems in liver fibrosis has been an issue that has taken great interest in recent years (72). Most NASH patients have been found with an upregulation of EC and apelin signals (73-75). EC are physiological ligands derived from arachidonic acid (AA) that interact with their receptors CB1 and CB2. It has been suggested that CB1 has an important role in NAFLD development and in diet-induced obesity mainly expressed in hepatocytes, LSECs, and HSCs (76) 


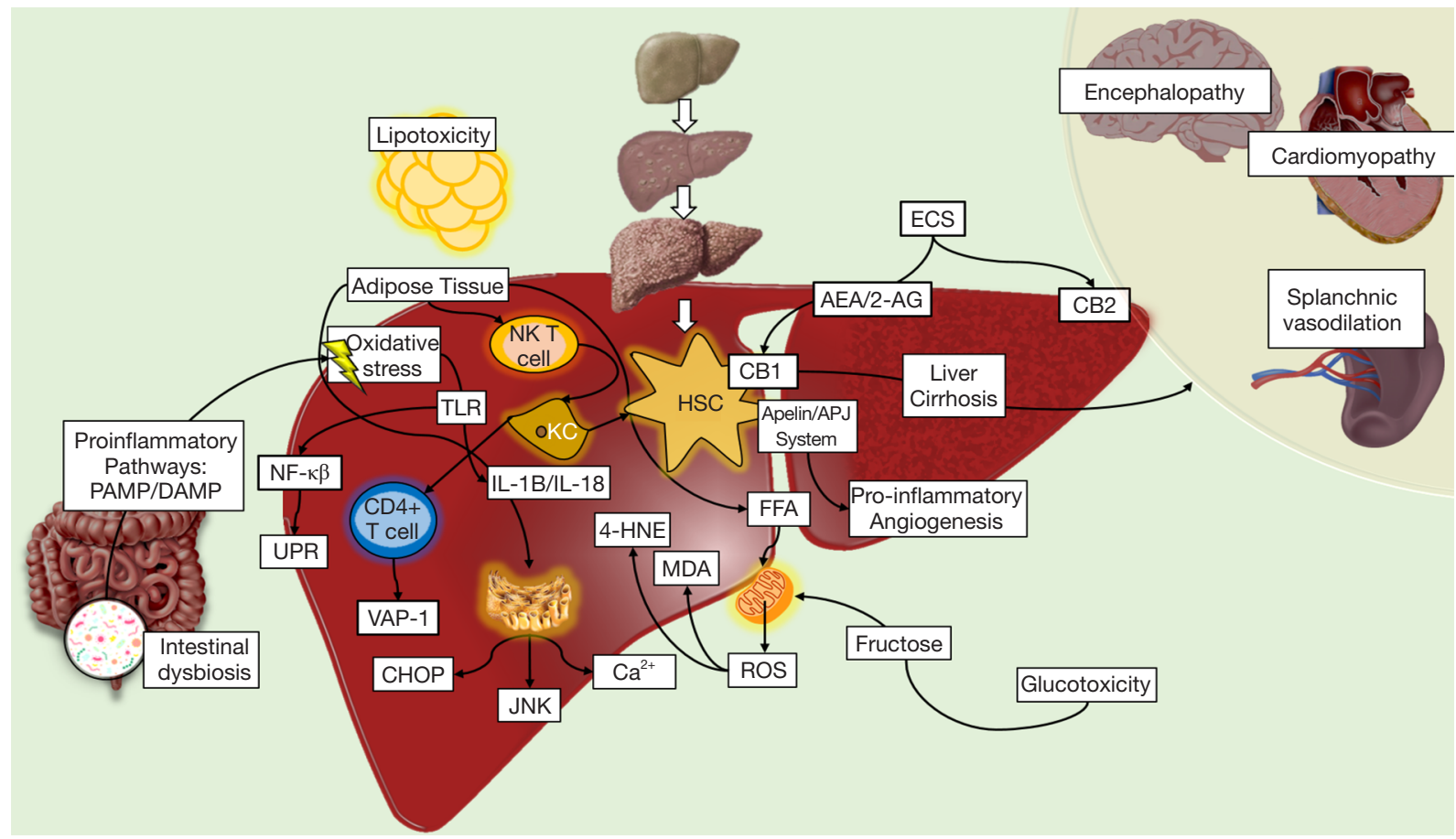

Figure 2 Hepatic stellate cells activation. Lipotoxicity plays an important role in producing dysfunction of the endoplasmic reticulum (ER) and mitochondria activating the CHOP and JNK pathways, altering calcium channels and favoring ROS formation. This will trigger the production of MDA and 4-HNE, respectively. Also, the activation of HSCs through KCs, which also induce the production of CD4+ T cell that increases VAP-1 concentrations, an amino oxidase expressed in the endothelium that is related to fibrosis severity. Other mechanisms are the ECS through the production of AEA and 2-AG that activates the CB1 receptor which further aggravates the state of fibrosis, as well as contributes to other systemic complications, while CB2 seems to have an opposite effect. In addition, the activation of the Apelin/APJ System promotes pro-inflammatory activity and angiogenesis that favors fibrosis development especially in early stages.

by seeing that its inactivation led to the apoptosis of HSCs and a decreased response to PDGF reducing the levels of TGF- $\beta$ expression and fibrosis (77) while CB2 was more expressed in HSCs and its upregulation was related with antifibrotic and anti-inflammatory effects (78). On the other hand, apelin is an endogenous ligand of an orphan receptor called angiotensin-like-receptor 1 (APJ). Apelin system has been related with important physiological events as EC system (72). In the liver, apelin is expressed in LSECs, HSCs, and leukocytes while in NASH pathogenesis, apelin has an important pro-fibrotic effect through partially mediating the fibrogenic effects of HSCs triggered by angiotensin II (AII) and endothelin 1 (ET-1) expression (79). Apelin has also been related with HSCs survival and synthesis of PDGF and type 1 collagen via ERK signaling (79). Additionally, apelin is an important angiogenic factor via endothelial APJ activation stimulating the expression of angiopoietin-1 in HSCs and favoring the hypoxic environment commonly seen in NASH, by the upregulation of the hypoxia-inducible factors (HIF-1,
HIF-2) with an important transcendence in fibrosis development $(80,81)$ (Figure 2).

Thus, we can summarize that HSCs are important components in the development of NASH. They are the major cells in fibrosis with the capacity to respond to KCs and hepatocytes stimulation. Even more, HSCs can release several transcriptional factors for itself and other cells in response to chronic liver injury (82-86).

\section{DCs}

In recent years DCs have emerged as an essential cell bridge for the connection between the innate and adaptive immune system response. DCs are a group of specialized hematopoietic cells that function as antigen presenting cells (APCs) in the liver. One of the most important properties that hepatic DCs (HDCs) possess, unlike other DCs in the human body, is the ability to preserve a tolerogenic atmosphere to maintain homeostasis in situations that are 
not so hostile for the liver (87). In a healthy environment DCs will present an immature phenotype characterized by a low capacity to endocytose antigens and to stimulate T-lymphocytes accompanied with a high production of IL10 and IL-27 promoting the differentiation of $\mathrm{CD}^{+} \mathrm{T}$ cells into regulatory $\mathrm{T}$ cells (Treg) maintaining the tolerance of self-antigens (88), however, as mentioned before, one of the main characteristics of NASH is the loss of the liver's tolerogenic environment, changing to a pro-inflammatory immunogenic phenotype. This will induce maturation of DCs favoring inflammation by the liberation of proinflammatory cytokines and inducing the adaptive immune response by an enhanced capacity to activate antigen specific $\mathrm{CD}^{+}{ }^{+} \mathrm{T}$ cells and $\mathrm{CD} 8^{+} \mathrm{T}$ cells (87). Nonetheless, the actual role of DCs in the pathogenesis of NASH is still a matter of debate, since DCs ablation studies have shown contrasting results depending on the experimental setting (88). Interestingly, the immune-stimulating and proinflammatory phenotype of HDCs seems to be associated with a high-lipid content in the cell. Also, depending on the differentiation pattern that HDCs express, it might determine the immunophenotypic response that these cells would achieve. In experimental animal models, myeloid HDCs (DC1) identified with the $\mathrm{CD}_{103^{+}}$marker appear to have a protective role in the liver by founding that the transference of $\mathrm{CD} 103^{+} \mathrm{CDC}_{1}$ to a $\mathrm{Batf}^{-/-}$deficient murine cohort reduced inflammatory monocyte recruitment, liver CCL2 expression and serum transaminases without affecting the extent of steatosis (89). By other side, DCCD40-ko mice $\left(\mathrm{CD} 40^{\mathrm{A} / \mathrm{f}} \mathrm{CD} 11 \mathrm{c}^{\mathrm{cre}}\right)$ subjected to obesity and NASH by feeding them with a high-fat diet (HFD) showed that CD40 expressing $\mathrm{CD} 11 \mathrm{c}^{+}$cells play a crucial role in protection against obesity-induced ectopic lipid storage and metabolic dysfunction, most likely via induction of Treg, however, during NASH, CD40 on CD11 $\mathrm{c}^{+}$cells contributes to liver inflammation (90). Additionally, NASH C57BL/6 mice fed with a methionine/choline-deficient (MCD)diet exhibited an overexpression of CD11 $\mathrm{c}^{\text {high }} / \mathrm{F} 4-80^{+}$DCs pool, but a reduced expression of $\mathrm{CD} 11 \mathrm{c}^{+} / \mathrm{MHCII}^{+} / \mathrm{B} 220^{+}$ plasmocytoid DCs (pDCs) and CD11 $\mathrm{c}^{+} / \mathrm{MHCII}^{+} / \mathrm{CD}^{2} \mathrm{a}^{+}$ lymphocytoid DCs (IDCs) (91).

In humans, we have shown that $\mathrm{CD} 11 \mathrm{C}^{+} \mathrm{cDC}_{2}$ were more elevated in obesity-induced NASH patients with fibrosis than those without fibrosis suggesting that $\mathrm{CD} 11 \mathrm{C}^{+} \mathrm{cDC}_{2}$ may have an important role in fibrosis development (92). In this scenario, an elegant transcriptional and immune profiling of patients with NASH was recently conducted showing interesting results (93). It was revealed that $\mathrm{cDC}_{2}$ were positively correlated with NASH progression while $\mathrm{cDC}_{1}$ and $\mathrm{pDC}$ were associated with a negative hepatic expression of genes involved in immune regulation and antigenic presentation making more understandable the role of these cells in NASH pathogenesis.

\section{Interplay between the innate and the adaptive immune response in NASH development}

As we have reviewed, an overnutrition state will generate an imbalance in AT storage and in the hepatic lipid metabolism promoting cellular stress, apoptosis and liver injury. The activation of KCs and the transdifferentiation of HSCs into myofibroblasts are the more important mechanisms within NASH and fibrosis development. However, the adaptive immune response will end up orchestrating the chronicity of inflammation and liver damage in NASH patients. In this context, DCs are responsible for the connection between the innate and adaptive response via major histocompatibility complex (MHC) class I and II molecules (94). Similarly, recent findings have described oxidative stress as one of the main triggers in stimulating adaptive immune response through a group of protein adducts with lipid peroxidation breakdown products, like malondialdehyde (MDA), malondialdehyde-acetaldehyde (MAA), and 4-hydroxynonenal, as well as phosphocholine (PC)containing oxidized phospholipids formally called oxidationspecific epitopes (OSEs) (95). OSEs are recognized by both innate and adaptive humoral immunity, including specific antibodies in an important number of systemic diseases (94). Evidence has suggested a protective mechanism of OSEspecific immunoglobulin $\mathrm{M}$ (IgM) antibodies in NASH in both animal and human models (96-98), while elevated titers of OSE-specific immunoglobulin G (IgG) antibodies are associated with the severity of lobular inflammation and a greater presence of intrahepatic B and T cell lymphocyte aggregates, in addition to being an independent fibrosis factor $(94,99,100)$ (Figure 3).

\section{Lymphocytes}

Lobular lymphocyte aggregates by $\mathrm{T}$ and $\mathrm{B}$ cells are a characteristic commonly found in patients with NASH. The size and prevalence of these lymphocyte aggregates correlated with the degree of lobular inflammation and fibrosis $(101,102) . \mathrm{CD}^{+} \mathrm{T}$ cells express CD44 and CD69 activation markers as well as an increase in the production of IFN- $\gamma$ and tumor necrosis factor superfamily member-14 (TNFSF14) 


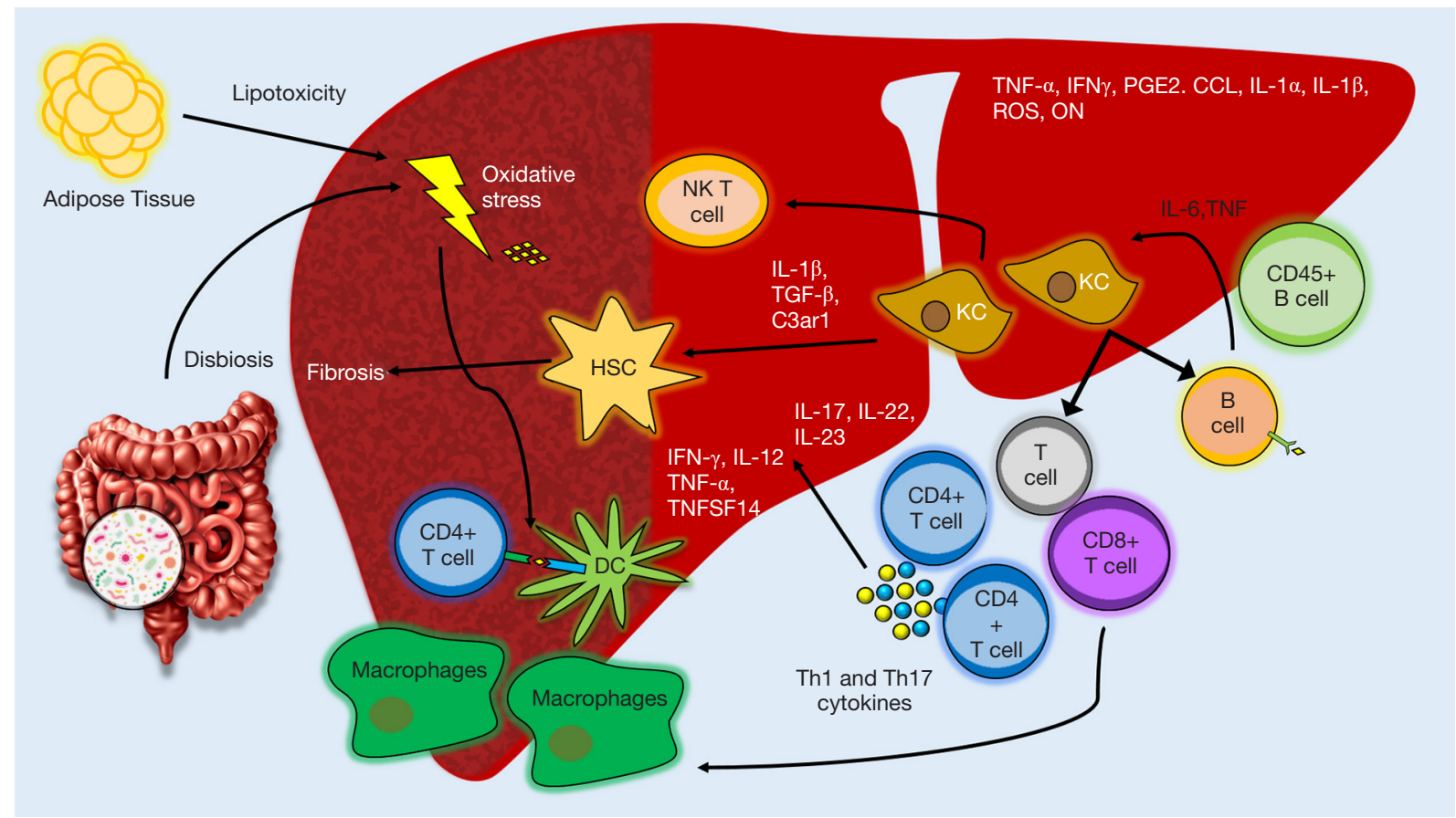

Figure 3 Oxidative stress in the progression of NASH. In the first instance, lipotoxicity and intestinal dysbiosis can produce an environment of oxidative stress, which contributes to the development of fibrosis through the activation of Kupffer cells (KCs) and hepatic stellate cells (HSCs), also, this stress generates a response from both the innate and adaptive immune system, through the production of oxidationspecific epitopes (OSEs) favoring the activation of KCs with the further released of pro-inflammatory cytokines and chemokines such as TNF $\alpha$, IFN $\gamma$, PGE2, CCL, IL-1 $\alpha$, IL-1 $\beta$, ROS, NO, TGF $\beta$ and C3ar1 activating natural killer T cells (NKT) and HSCs. In addition, KCs will enhance the differentiation of both $\mathrm{T}$ and $\mathrm{B}$ cells. CD4+ T cells will polarize into Th1 and Th17 cells promoting the activation of macrophages and establishing lymphocytic infiltrates aggravating the pro-inflammatory state. On the other hand, CD45+ B cells will express anti-OSE IgG enhancing the activation of CD4+ T cells.

indicating that lymphocyte aggregates are functionally active. Moreover, in addition to the activation mediated by DCs, it has recently been demonstrated through the use of coculture systems, that KCs promote adult liver hematopoietic stem and progenitor cells (HSPCs) to primarily generate $\mathrm{T}$ cells and $\mathrm{B}$ cells via intercellular cell adhesion molecule-1 (ICAM1). A blockade in ICAM-1 impaired the adhesion, expansion, and differentiation of HSPCs suggesting another important induction-pathway of adaptive immune response (103). Also, the vascular adhesion protein 1 (VAP-1) is a membranebound amine oxidase expressed in liver endothelium that supports lymphocytes adhesion and transendothelial migration across LSECs in many inflammatory diseases (11). VAP-1 catalyzes the oxidative deamination of primary amines resulting in the generation of aldehyde, ammonia and hydrogen peroxide (104). These products activate the secretion of NFкB-dependent chemokines and the expression of adhesion molecules in the liver endothelium $(105,106)$.
Furthermore, Weston et al. found that the soluble form of VAP-1 (sVAP-1) in the liver was markedly elevated in NAFLD patients when compared to controls and in VAP1-deficient mice $\left(\mathrm{Aoc}^{-/-}\right.$) they probed that VAP-1 promotes NASH progression by observing that a poor expression of VAP-1 protected against the accumulation of intrahepatic $\mathrm{T}$ cells, NKT cells, and myeloid subsets (11) (Figure 2).

\section{T cells}

In a healthy host, DCs are responsible for expressing negative regulators for $\mathrm{T}$ cells response such as cytotoxic T lymphocyte antigen 4 (CTLA-4), IL-10, TGF- $\beta$ and programmed death-ligand 1 (PD-L1) favoring the polarization of $\mathrm{CD}^{+} \mathrm{T}$ cells into Treg maintaining the tolerance to self-antigens and avoiding an excessive effector-T-cell activation and subsequent tissue damage during infection-induced immune responses $(107,108)$. 
Interestingly, in NAFLD-induced animal models, a reduced number of hepatic Treg cells has been found $(109,110)$. Apparently, this reduction is due to local ROS-induced apoptosis of Treg cells. Moreover, an adoptive transfer of Treg cells attenuated hepatic inflammation and decrease hepatic TNF- $\alpha$ expression (110).

Nonetheless, within NASH development DCs perform the antigen presentation of MHC class II molecules to the specialized $\mathrm{CD}^{+}{ }^{+} \mathrm{T}$ cell receptor (TCR) and the co-receptor CD3 favoring the polarization into T helper-1 (Th1) and T helper-17 (Th17) (94). Th1 cells are proinflammatory cells that express the transcription factor T-bet producing IFN- $\gamma$, IL-12, TNF- $\alpha$ and TNFSF14 (108). Th1 cells have been involved in AT inflammation associated with an overexpression of leptin in HFD-animal models of obesity (111-113). In humans, a couple of studies have demonstrated increased levels of Th1 cells in peripheral blood of NASH patients $(114,115)$ and in a MCD-diet murine model of NASH an increase in liver Th1 cells was found only in advanced stages of the disease speculating a possible relation with the fibrosis development (116).

As mentioned early, the other important phenotype of $\mathrm{CD}^{+}{ }^{+} \mathrm{T}$ cells in NASH is Th17 cells. These are proinflammatory cells that express the transcription factor retinoic acid receptor-related orphan receptor $\gamma \mathrm{t}(\mathrm{ROR} \gamma \mathrm{t})$, as well as the signal transducer and activator of transcription 3 (STAT3). Their main action is to reinforce the adaptive immune response against external agents by the expression of IL-17, IL-22 and IL-23. Interestingly, the progression of NAFLD to NASH has been associated with the liver accumulation of Th17 cells (117). Also, some studies have found a Th-17-induced liver inflammation through an accumulation of macrophages via IL-17-dependent upregulation of CXCL-10 (118,119). Similarly, Rolla et al. suggested that lipotoxic effects of FFAs are exacerbated in the presence of IL-17 in a JNK- dependent manner (116).

On the other hand, CD $8^{+} \mathrm{T}$ cells are the special cytotoxic cells aimed to eliminate infected or malignant cells. They are mediated by the recognition of MHC class I molecules by APCs and until today, their role in NASH pathogenesis is still not clear. Nishimura et al. have shown that $\mathrm{CD} 8^{+} \mathrm{T}$ cells are essential for macrophage recruitment and AT inflammation (120). Evidence has also confirmed an important liver infiltration of $\mathrm{CD} 8^{+} \mathrm{T}$ cells in human and animal models of NAFLD (121-123) while the blockade of these cells with anti-CD8 antibodies result in a decrease in liver steatosis, liver inflammation and transaminase levels $(9,121)$, as well as reduced HSCs activation (9). Similarly, in the elegant study of Haas et al. (93) they suggest that intrahepatic accumulation of $\mathrm{CD} 8^{+} \mathrm{T}$ cells and a disruption in $\mathrm{cDC} 1 / \mathrm{cDC} 2$ ratio were the main immune hallmark in $\mathrm{NASH}$ activity and progression.

\section{B cells}

Some observational studies in obesity-induced mouse models have found an association between $\mathrm{CD} 45^{+} \mathrm{B}$-cell infiltrates in AT and IR, systemic inflammation, increased production of proinflammatory cytokines and T-cells and macrophages activation (124-126). Few studies have focused on determine the role of intrahepatic B cells in NAFLD. In this scenario, Zhang et al. (127) found that $\mathrm{CD} 5^{+}$intrahepatic B (IHB) cells were significantly higher in NAFLD patients than controls, expressing higher levels of IL-6, and TNF- $\alpha$. Moreover, IHB cells enhanced the activation of $\mathrm{CD}^{+} \mathrm{T}$ cells promoting the polarization into Th1 cells in the NAFLD group (75). Likewise, Bruzzì et al. showed in a mouse-model of NAFLD that the onset of NASH was characterized by hepatic B2 cells maturation to plasma B cells and by an elevation in circulating anti-OSE IgG titers. The $\mathrm{B}$ cells responses preceded $\mathrm{T}$ cells activation and the up-regulation of the hepatic expression of B-cell Activating Factor (BAFF). The selective B2-cells depletion in mice prevented the plasma $\mathrm{B}$ cells maturation and the Th1 polarization of $\mathrm{CD}^{+}{ }^{+} \mathrm{T}$-cells accompanied with a milder steatohepatitis and fibrosis (101).

\section{Conclusions}

The liver is an extraordinary organ with the ability to preserve a tolerogenic environment despite the multiple harmful agents derived from the enterohepatic circulation and with the capacity of recover from acute liver injury in most of the cases. Nonetheless, in NASH the hepatic tissue repair capacity is exceeded by the accumulation of cytotoxic lipids derived from an overnutrition diet triggering cells dysfunction and the activation of the innate immune response with the consequent development of fibrosis. Recent findings have also suggested that $T$ and $B$ cells are essential for the maintenance of the liver inflammation and the fibrosis development by inducing the expression of cytokines and chemokines important for the KCs polarization and the HDCs activation. Therefore, the study of both immune responses in NASH is crucial in order to implement new therapeutic options for the near future. 


\section{Acknowledgments}

Funding: This study was partly supported by a grant from Medica Sur Clinic and Foundation.

\section{Footnote}

Provenance and Peer Review: This article was commissioned by the Guest Editor (Nahum Méndez-Sánchez) for the series "Liver News Column" published in Annals of Translational Medicine. The article was sent for external peer review organized by the Guest Editor and the editorial office.

Conflicts of Interest: The series "Liver News Column" was commissioned by the editorial office without any funding or sponsorship. NMS served as the unpaid the unpaid Guest Editor of the series. The other authors have no other conflicts of interest to declare.

Ethical Statement: The authors are accountable for all aspects of the work in ensuring that questions related to the accuracy or integrity of any part of the work are appropriately investigated and resolved.

Open Access Statement: This is an Open Access article distributed in accordance with the Creative Commons Attribution-NonCommercial-NoDerivs 4.0 International License (CC BY-NC-ND 4.0), which permits the noncommercial replication and distribution of the article with the strict proviso that no changes or edits are made and the original work is properly cited (including links to both the formal publication through the relevant DOI and the license). See: https://creativecommons.org/licenses/by-nc-nd/4.0/.

\section{References}

1. Tanaka M, Miyajima A. Liver regeneration and fibrosis after inflammation. Inflamm Regen 2016;36:19.

2. Nakamura T, Mizuno S. The discovery of hepatocyte growth factor (HGF) and its significance for cell biology, life sciences and clinical medicine. Proc Jpn Acad, Ser B, Phys Biol Sci 2010;86:588-610.

3. Fausto N, Campbell JS, Riehle KJ. Liver regeneration. J Hepatol 2012;57:692-4.

4. Guicciardi ME, Gores GJ. Apoptosis: a mechanism of acute and chronic liver injury. Gut 2005;54:1024-33.

5. Böhm F, Köhler UA, Speicher T, et al. Regulation of liver regeneration by growth factors and cytokines. $\mathrm{EMBO} \mathrm{Mol}$
Med 2010;2:294-305.

6. Wick G, Backovic A, Rabensteiner E, et al. The immunology of fibrosis: innate and adaptive responses. Trends Immunol 2010;31:110-9.

7. Méndez-Sánchez N, Zamarripa-Dorsey F, Panduro A, et al. Current trends of liver cirrhosis in Mexico: Similitudes and differences with other world regions. World J Clin Cases 2018;6:922-30.

8. Méndez-Sánchez N, Villa AR, Chávez-Tapia NC, et al. Trends in liver disease prevalence in Mexico from 2005 to 2050 through mortality data. Ann Hepatol 2005;4:52-5.

9. Wolf MJ, Adili A, Piotrowitz K, et al. Metabolic activation of intrahepatic CD8+ T cells and NKT cells causes nonalcoholic steatohepatitis and liver cancer via cross-talk with hepatocytes. Cancer Cell 2014;26:549-64.

10. Herrero-Cervera A, Vinué Á, Burks DJ, et al. Genetic inactivation of the LIGHT (TNFSF14) cytokine in mice restores glucose homeostasis and diminishes hepatic steatosis. Diabetologia 2019;62:2143-57.

11. Weston CJ, Shepherd EL, Claridge LC, et al. Vascular adhesion protein-1 promotes liver inflammation and drives hepatic fibrosis. J Clin Invest 2015;125:501-20.

12. Grohmann M, Wiede F, Dodd GT, et al. Obesity Drives STAT-1-Dependent NASH and STAT-3-Dependent HCC. Cell 2018;175:1289-306.e20.

13. Almeda-Valdes P, Aguilar-Olivos N, Uribe M, et al. Common features of the metabolic syndrome and nonalcoholic fatty liver disease. Rev Recent Clin Trials 2014;9:148-58.

14. Sanders FWB, Griffin JL. De novo lipogenesis in the liver in health and disease: more than just a shunting yard for glucose. Biol Rev Camb Philos Soc 2016;91:452-68.

15. Mendez-Sanchez N, Cruz-Ramon VC, Ramirez-Perez OL, et al. New Aspects of Lipotoxicity in Nonalcoholic Steatohepatitis. Int J Mol Sci 2018. doi: 10.3390/ ijms19072034.

16. Beaven SW, Matveyenko A, Wroblewski K, et al. Reciprocal regulation of hepatic and adipose lipogenesis by liver $\mathrm{X}$ receptors in obesity and insulin resistance. Cell Metab 2013;18:106-17.

17. Jump DB, Lytle KA, Depner CM, et al. Omega-3 polyunsaturated fatty acids as a treatment strategy for nonalcoholic fatty liver disease. Pharmacol Ther 2018;181:108-25.

18. Lancaster GI, Langley KG, Berglund NA, et al. Evidence that TLR4 Is Not a Receptor for Saturated Fatty Acids but Mediates Lipid-Induced Inflammation by Reprogramming Macrophage Metabolism. Cell 
Metab 2018;27:1096-110.e5.

19. Pierantonelli I, Svegliati-Baroni G. Nonalcoholic Fatty Liver Disease: Basic Pathogenetic Mechanisms in the Progression From NAFLD to NASH. Transplantation 2019;103:e1-13.

20. Ter Horst KW, Serlie MJ. Fructose Consumption, Lipogenesis, and Non-Alcoholic Fatty Liver Disease. Nutrients 2017. doi: 10.3390/nu9090981.

21. Fuchs M, Sanyal AJ. Lipotoxicity in NASH. J Hepatol 2012;56:291-3.

22. Sunny NE, Bril F, Cusi K. Mitochondrial Adaptation in Nonalcoholic Fatty Liver Disease: Novel Mechanisms and Treatment Strategies. Trends Endocrinol Metab 2017;28:250-60.

23. Patterson RE, Kalavalapalli S, Williams CM, et al. Lipotoxicity in steatohepatitis occurs despite an increase in tricarboxylic acid cycle activity. Am J Physiol Endocrinol Metab 2016;310:E484-94.

24. Stanger BZ. Cellular homeostasis and repair in the mammalian liver. Annu Rev Physiol 2015;77:179-200.

25. Benedict $M$, Zhang X. Non-alcoholic fatty liver disease: An expanded review. World J Hepatol 2017;9:715-32.

26. Hu P, Han Z, Couvillon AD, et al. Autocrine tumor necrosis factor alpha links endoplasmic reticulum stress to the membrane death receptor pathway through IRE1alphamediated NF-kappaB activation and down-regulation of TRAF2 expression. Mol Cell Biol 2006;26:3071-84.

27. Tam AB, Mercado EL, Hoffmann A, et al. ER stress activates NF- $\mathrm{KB}$ by integrating functions of basal IKK activity, IRE1 and PERK. PLoS ONE 2012;7:e45078.

28. Urano F, Wang X, Bertolotti A, et al. Coupling of stress in the ER to activation of JNK protein kinases by transmembrane protein kinase IRE1. Science 2000;287:664-6.

29. Tabas I, Ron D. Integrating the mechanisms of apoptosis induced by endoplasmic reticulum stress. Nat Cell Biol 2011;13:184-90.

30. Hetz C, Bernasconi P, Fisher J, et al. Proapoptotic BAX and BAK modulate the unfolded protein response by a direct interaction with IRE1alpha. Science 2006;312:572-6.

31. Honda F, Hiramatsu A, Hyogo H, et al. Evaluation of glycemic variability in chronic liver disease patients with type 2 diabetes mellitus using continuous glucose monitoring. PLoS ONE 2018;13:e0195028.

32. Simões ICM, Fontes A, Pinton P, et al. Mitochondria in non-alcoholic fatty liver disease. Int J Biochem Cell Biol 2018;95:93-9.

33. Yin X, Zheng F, Pan Q, et al. Glucose fluctuation increased hepatocyte apoptosis under lipotoxicity and the involvement of mitochondrial permeability transition opening. J Mol Endocrinol 2015;55:169-81.

34. Mantena SK, Vaughn DP, Andringa KK, et al. High fat diet induces dysregulation of hepatic oxygen gradients and mitochondrial function in vivo. Biochem J 2009;417:183-93.

35. Pessayre D, Berson A, Fromenty B, et al. Mitochondria in steatohepatitis. Semin Liver Dis 2001;21:57-69.

36. Carter-Kent C, Zein NN, Feldstein AE. Cytokines in the pathogenesis of fatty liver and disease progression to steatohepatitis: implications for treatment. Am J Gastroenterol 2008;103:1036-42.

37. Rodrigues PM, Afonso MB, Simão AL, et al. miR-21 ablation and obeticholic acid ameliorate nonalcoholic steatohepatitis in mice. Cell Death Dis 2017;8:e2825.

38. Garcia-Martinez I, Santoro N, Chen Y, et al. Hepatocyte mitochondrial DNA drives nonalcoholic steatohepatitis by activation of TLR9. J Clin Invest 2016;126:859-64.

39. Gan LT, Van Rooyen DM, Koina ME, et al. Hepatocyte free cholesterol lipotoxicity results from JNK1-mediated mitochondrial injury and is HMGB1 and TLR4dependent. J Hepatol 2014;61:1376-84.

40. Begriche K, Igoudjil A, Pessayre D, et al. Mitochondrial dysfunction in NASH: causes, consequences and possible means to prevent it. Mitochondrion 2006;6:1-28.

41. Heymann F, Tacke F. Immunology in the liver--from homeostasis to disease. Nat Rev Gastroenterol Hepatol 2016;13:88-110.

42. Liu XL, Pan Q, Cao HX, et al. Lipotoxic HepatocyteDerived Exosomal miR-192-5p Activates Macrophages via Rictor/Akt/FoxO1 Signaling in NAFLD. Hepatology 2019. [Epub ahead of print].

43. Hirsova P, Ibrahim SH, Krishnan A, et al. Lipid-Induced Signaling Causes Release of Inflammatory Extracellular Vesicles From Hepatocytes. Gastroenterology 2016;150:956-67.

44. Kolios G, Valatas V, Kouroumalis E. Role of Kupffer cells in the pathogenesis of liver disease. World J Gastroenterol 2006;12:7413-20.

45. Dixon LJ, Barnes M, Tang H, et al. Kupffer cells in the liver. Compr Physiol 2013;3:785-97.

46. Handa P, Vemulakonda A, Kowdley KV, et al. Mitochondrial DNA from hepatocytes as a ligand for TLR9: Drivers of nonalcoholic steatohepatitis? World J Gastroenterol 2016;22:6965-71.

47. Almeda-Valdes P, Altamirano-Barrera A, Méndez-Sánchez N. Insights in non-alcoholic fatty liver disease pathophysiology with lipidomic analyses. Ann Hepatol 2015;14:567-9. 
48. Roh YS, Seki E. Toll-like receptors in alcoholic liver disease, non-alcoholic steatohepatitis and carcinogenesis. J Gastroenterol Hepatol 2013;28 Suppl 1:38-42.

49. Cai J, Zhang XJ, Li H. The Role of Innate Immune Cells in Nonalcoholic Steatohepatitis. Hepatology 2019;70:1026-37.

50. Duque Correa MA, Rojas López M. Activación alternativa del macrófago: La diversidad en las respuestas de una célula de la inmunidad innata ante la complejidad de los eventos de su ambiente. Inmunologia 2007;26:73-86.

51. Ramadori P, Kroy D, Streetz KL. Immunoregulation by lipids during the development of non-alcoholic steatohepatitis. Hepatobiliary Surg Nutr 2015;4:11-23.

52. Batista-Gonzalez A, Vidal R, Criollo A, et al. New Insights on the Role of Lipid Metabolism in the Metabolic Reprogramming of Macrophages. Front Immunol 2020;10:2993.

53. Choe JY, Kim SK. Quercetin and Ascorbic Acid Suppress Fructose-Induced NLRP3 Inflammasome Activation by Blocking Intracellular Shuttling of TXNIP in Human Macrophage Cell Lines. Inflammation 2017;40:980-94.

54. Freemerman AJ, Johnson AR, Sacks GN, et al. Metabolic reprogramming of macrophages: glucose transporter 1 (GLUT1)-mediated glucose metabolism drives a proinflammatory phenotype. J Biol Chem 2014;289:7884-96.

55. Baffy G. Kupffer cells in non-alcoholic fatty liver disease: the emerging view. J Hepatol 2009;51:212-23.

56. Tosello-Trampont AC, Landes SG, Nguyen V, et al. Kuppfer cells trigger nonalcoholic steatohepatitis development in diet-induced mouse model through tumor necrosis factor- $\alpha$ production. J Biol Chem 2012;287:40161-72.

57. Stienstra R, Saudale F, Duval C, et al. Kupffer cells promote hepatic steatosis via interleukin-1beta-dependent suppression of peroxisome proliferator-activated receptor alpha activity. Hepatology 2010;51:511-22.

58. Miura K, Yang L, van Rooijen N, et al. Hepatic recruitment of macrophages promotes nonalcoholic steatohepatitis through CCR2. Am J Physiol Gastrointest Liver Physiol 2012;302:G1310-21.

59. Wan J, Benkdane M, Teixeira-Clerc F, et al. M2 Kupffer cells promote M1 Kupffer cell apoptosis: a protective mechanism against alcoholic and nonalcoholic fatty liver disease. Hepatology 2014;59:130-42.

60. Spann NJ, Glass CK. Sterols and oxysterols in immune cell function. Nat Immunol 2013;14:893-900.

61. Sag D, Carling D, Stout RD, et al. Adenosine 5'-monophosphate-activated protein kinase promotes macrophage polarization to an anti-inflammatory functional phenotype. J Immunol 2008;181:8633-41.

62. Han J, Zhang X, Lau JK, et al. Bone marrow-derived macrophage contributes to fibrosing steatohepatitis through activating hepatic stellate cells. J Pathol 2019;248:488-500.

63. Wilson CL, Mann J, Walsh M, et al. Quiescent Hepatic Stellate Cells Functionally Contribute to the Hepatic Innate Immune Response via TLR3. PLoS One 2014;9:e83391.

64. Coombes JD, Choi SS, Swiderska-Syn M, et al. Osteopontin is a proximal effector of leptin-mediated non-alcoholic steatohepatitis (NASH) fibrosis. Biochim Biophys Acta 2016;1862:135-44.

65. Polyzos SA, Aronis KN, Kountouras J, et al. Circulating leptin in non-alcoholic fatty liver disease: a systematic review and meta-analysis. Diabetologia 2016;59:30-43.

66. Saxena NK, Anania FA. Adipocytokines and hepatic fibrosis. Trends Endocrinol Metab 2015;26:153-61.

67. Liu C, Tao Q, Sun M, et al. Kupffer cells are associated with apoptosis, inflammation and fibrotic effects in hepatic fibrosis in rats. Lab Invest 2010;90:1805-16.

68. Hetherington AM, Sawyez CG, Zilberman E, et al. Differential Lipotoxic Effects of Palmitate and Oleate in Activated Human Hepatic Stellate Cells and Epithelial Hepatoma Cells. Cell Physiol Biochem 2016;39:1648-62.

69. Ioannou GN. The Role of Cholesterol in the Pathogenesis of NASH. Trends Endocrinol Metab 2016;27:84-95.

70. Chu X, Jin Q, Chen H, et al. CCL20 is up-regulated in non-alcoholic fatty liver disease fibrosis and is produced by hepatic stellate cells in response to fatty acid loading. J Transl Med 2018;16:108.

71. Kim B-M, Abdelfattah AM, Vasan R, et al. Hepatic stellate cells secrete Cc15 to induce hepatocyte steatosis. Sci Rep 2018;8:7499.

72. Melgar-Lesmes P, Perramon M, Jiménez W. Roles of the Hepatic Endocannabinoid and Apelin Systems in the Pathogenesis of Liver Fibrosis. Cells 2019. doi: 10.3390/ cells8111311.

73. Mendez-Sanchez N, Zamora-Valdes D, Pichardo-Bahena $\mathrm{R}$, et al. Endocannabinoid receptor CB2 in nonalcoholic fatty liver disease. Liver Int 2007;27:215-9.

74. Fernández-Rodriguez CM, Romero J, Petros TJ, et al. Circulating endogenous cannabinoid anandamide and portal, systemic and renal hemodynamics in cirrhosis. Liver Int 2004;24:477-83.

75. Principe A, Melgar-Lesmes P, Fernández-Varo G, et al. The hepatic apelin system: a new therapeutic target for liver disease. Hepatology 2008;48:1193-201. 
76. Osei-Hyiaman D, DePetrillo M, Pacher P, et al. Endocannabinoid activation at hepatic CB1 receptors stimulates fatty acid synthesis and contributes to dietinduced obesity. J Clin Invest 2005;115:1298-305.

77. Teixeira-Clerc F, Belot MP, Manin S, et al. Beneficial paracrine effects of cannabinoid receptor 2 on liver injury and regeneration. Hepatology 2010;52:1046-59.

78. Reichenbach V, Ros J, Fernández-Varo G, et al. Prevention of fibrosis progression in CCl4-treated rats: role of the hepatic endocannabinoid and apelin systems. J Pharmacol Exp Ther 2012;340:629-37.

79. Melgar-Lesmes P, Casals G, Pauta M, et al. Apelin mediates the induction of profibrogenic genes in human hepatic stellate cells. Endocrinology 2010;151:5306-14.

80. Rosmorduc O, Housset C. Hypoxia: a link between fibrogenesis, angiogenesis, and carcinogenesis in liver disease. Semin Liver Dis 2010;30:258-70.

81. Cannito S, Paternostro C, Busletta C, et al. Hypoxia, hypoxia-inducible factors and fibrogenesis in chronic liver diseases. Histol Histopathol 2014;29:33-44.

82. Puche JE, Lee YA, Jiao J, et al. A novel murine model to deplete hepatic stellate cells uncovers their role in amplifying liver damage in mice. Hepatology 2013;57:339-50.

83. Tang L, Tanaka Y, Marumo F, et al. Phenotypic change in portal fibroblasts in biliary fibrosis. Liver 1994;14:76-82.

84. Herbst H, Frey A, Heinrichs O, et al. Heterogeneity of liver cells expressing procollagen types I and IV in vivo. Histochem Cell Biol 1997;107:399-409.

85. Cassiman D, Libbrecht L, Desmet V, et al. Hepatic stellate cell/myofibroblast subpopulations in fibrotic human and rat livers. J Hepatol 2002;36:200-9.

86. Fausther M, Goree JR, Lavoie ÉG, et al. Establishment and characterization of rat portal myofibroblast cell lines. PLoS One 2015;10:e121161.

87. Lukacs-Kornek V, Schuppan D. Dendritic cells in liver injury and fibrosis: Shortcomings and promises. J Hepatol 2013;59:1124-6.

88. Bernsmeier C, Albano E. Liver dendritic cells and NAFLD evolution: A remaining open issue. J Hepatol 2017;66:1120-2.

89. Heier EC, Meier A, Julich-Haertel H, et al. Murine CD103+ dendritic cells protect against steatosis progression towards steatohepatitis. J Hepatol 2017;66:1241-50.

90. Aarts S, Reiche M, den Toom M, et al. Depletion of CD40 on CD11c+ cells worsens the metabolic syndrome and ameliorates hepatic inflammation during NASH. Sci Rep 2019;9:14702.

91. Sutti S, Locatelli I, Bruzzì S, et al. CX3CR1-expressing inflammatory dendritic cells contribute to the progression of steatohepatitis. Clin Sci 2015;129:797-808.

92. Ramírez-Pérez OL, Barranco-Fragoso B, PichardoBahena R, et al. The role of dendritic cells in different stages of non-alcoholic fatty liver disease. J Hepatol 2018;68:S363-4.

93. Haas JT, Vonghia L, Mogilenko DA, et al. Transcriptional network analysis implicates altered hepatic immune function in NASH development and resolution. Nat Metab 2019;1:604-14.

94. Sutti S, Albano E. Adaptive immunity: an emerging player in the progression of NAFLD. Nat Rev Gastroenterol Hepatol 2020;17:81-92.

95. Chou MY, Fogelstrand L, Hartvigsen K, et al. Oxidationspecific epitopes are dominant targets of innate natural antibodies in mice and humans. J Clin Invest 2009;119:1335-49.

96. Binder CJ. Natural IgM antibodies against oxidationspecific epitopes. J Clin Immunol 2010;30 Suppl 1:S56-60.

97. Bieghs V, van Gorp PJ, Walenbergh SMA, et al. Specific immunization strategies against oxidized lowdensity lipoprotein: a novel way to reduce nonalcoholic steatohepatitis in mice. Hepatology 2012;56:894-903.

98. Hendrikx T, Watzenböck ML, Walenbergh SMA, et al. Low levels of IgM antibodies recognizing oxidationspecific epitopes are associated with human non-alcoholic fatty liver disease. BMC Med 2016;14:107.

99. Albano E, Mottaran E, Vidali M, et al. Immune response towards lipid peroxidation products as a predictor of progression of non-alcoholic fatty liver disease to advanced fibrosis. Gut 2005;54:987-93.

100. Nobili V, Parola M, Alisi A, et al. Oxidative stress parameters in paediatric non-alcoholic fatty liver disease. Int J Mol Med 2010;26:471-6.

101. Bruzzì S, Sutti S, Giudici G, et al. B2-Lymphocyte responses to oxidative stress-derived antigens contribute to the evolution of nonalcoholic fatty liver disease (NAFLD). Free Radic Biol Med 2018;124:249-59.

102. Pitzalis C, Jones GW, Bombardieri M, et al. Ectopic lymphoid-like structures in infection, cancer and autoimmunity. Nat Rev Immunol 2014;14:447-62 .

103. Meng D, Qin Y, Lu N, et al. Kupffer Cells Promote the Differentiation of Adult Liver Hematopoietic Stem and Progenitor Cells into Lymphocytes via ICAM-1 and LFA1 Interaction. Stem Cells Int 2019;2019:4848279.

104.Lyles GA. Mammalian plasma and tissue-bound semicarbazide-sensitive amine oxidases: biochemical, pharmacological and toxicological aspects. Int J Biochem 
Cell Biol 1996;28:259-74.

105.Lalor PF, Sun PJ, Weston CJ, et al. Activation of vascular adhesion protein-1 on liver endothelium results in an NF-kappaB-dependent increase in lymphocyte adhesion. Hepatology 2007;45:465-74.

106.Jalkanen S, Karikoski M, Mercier N, et al. The oxidase activity of vascular adhesion protein-1 (VAP-1) induces endothelial E- and P-selectins and leukocyte binding. Blood 2007;110:1864-70.

107.Laurent S, Carrega P, Saverino D, et al. CTLA-4 is expressed by human monocyte-derived dendritic cells and regulates their functions. Hum Immunol 2010;71:934-41.

108. Van Herck MA, Weyler J, Kwanten WJ, et al. The Differential Roles of T Cells in Non-alcoholic Fatty Liver Disease and Obesity. Front Immunol 2019;10:82.

109. Ma C, Kesarwala AH, Eggert T, et al. NAFLD causes selective CD4(+) T lymphocyte loss and promotes hepatocarcinogenesis. Nature 2016;531:253-7.

110.Ma X, Hua J, Mohamood AR, et al. A high-fat diet and regulatory $\mathrm{T}$ cells influence susceptibility to endotoxininduced liver injury. Hepatology 2007;46:1519-29.

111. Rocha VZ, Folco EJ, Sukhova G, et al. Interferon-gamma, a Th1 cytokine, regulates fat inflammation: a role for adaptive immunity in obesity. Circ Res 2008;103:467-76.

112.Hong CP, Park A, Yang BG, et al. Gut-Specific Delivery of T-Helper 17 Cells Reduces Obesity and Insulin Resistance in Mice. Gastroenterology 2017;152:1998-2010.

113. Khan IM, Dai Perrard XY, Perrard JL, et al. Attenuated adipose tissue and skeletal muscle inflammation in obese mice with combined CD4+ and CD8+ T cell deficiency. Atherosclerosis 2014;233:419-28.

114. Ferreyra Solari NE, Inzaugarat ME, Baz P, et al. The role of innate cells is coupled to a Th1-polarized immune response in pediatric nonalcoholic steatohepatitis. J Clin Immunol 2012;32:611-21.

115. Inzaugarat ME, Ferreyra Solari NE, Billordo LA, et al. Altered phenotype and functionality of circulating immune cells characterize adult patients with nonalcoholic steatohepatitis. J Clin Immunol 2011;31:1120-30.

116. Rolla S, Alchera E, Imarisio C, et al. The balance between IL-17 and IL-22 produced by liver-infiltrating T-helper cells critically controls NASH development in mice. Clin Sci 2016;130:193-203.

117. Rau M, Schilling AK, Meertens J, et al. Progression from Nonalcoholic Fatty Liver to Nonalcoholic Steatohepatitis Is Marked by a Higher Frequency of Th17 Cells in the Liver and an Increased Th17/Resting Regulatory T Cell Ratio in Peripheral Blood and in the Liver. J Immunol
2016;196:97-105.

118. Giles DA, Moreno-Fernandez ME, Stankiewicz TE, et al. Regulation of Inflammation by IL-17A and IL17F Modulates Non-Alcoholic Fatty Liver Disease Pathogenesis. PLoS One 2016;11:e0149783.

119.Xu R, Tao A, Zhang S, et al. Neutralization of interleukin-17 attenuates high fat diet-induced nonalcoholic fatty liver disease in mice. Acta Biochim Biophys Sin (Shanghai) 2013;45:726-33.

120. Nishimura S, Manabe I, Nagasaki M, et al. CD8+ effector $\mathrm{T}$ cells contribute to macrophage recruitment and adipose tissue inflammation in obesity. Nat Med 2009;15:914-20.

121. Bhattacharjee J, Kirby M, Softic S, et al. Hepatic Natural Killer T-cell and CD8+ T-cell Signatures in Mice with Nonalcoholic Steatohepatitis. Hepatol Commun 2017;1:299-310.

122. Ghazarian M, Revelo XS, Nøhr MK, et al. Type I Interferon Responses Drive Intrahepatic T cells to Promote Metabolic Syndrome. Sci Immunol 2017. doi: 10.1126/sciimmunol.aai7616.

123.Gadd VL, Skoien R, Powell EE, et al. The portal inflammatory infiltrate and ductular reaction in human nonalcoholic fatty liver disease. Hepatology 2014;59:1393-405.

124. McLaughlin T, Ackerman SE, Shen L, et al. Role of innate and adaptive immunity in obesity-associated metabolic disease. J Clin Invest 2017;127:5-13.

125.DeFuria J, Belkina AC, Jagannathan-Bogdan M, et al. $\mathrm{B}$ cells promote inflammation in obesity and type 2 diabetes through regulation of T-cell function and an inflammatory cytokine profile. Proc Natl Acad Sci USA 2013;110:5133-8.

126. Winer DA, Winer S, Shen L, et al. B cells promote insulin resistance through modulation of $\mathrm{T}$ cells and production of pathogenic IgG antibodies. Nat Med 2011;17:610-7.

127.Zhang F, Jiang WW, Li X, et al. Role of intrahepatic B cells in non-alcoholic fatty liver disease by secreting proinflammatory cytokines and regulating intrahepatic $\mathrm{T}$ cells: Role of B cells in NAFLD. J Dig Dis 2016;17:464-74.

Cite this article as: Méndez-Sánchez N, Valencia-Rodríguez A, Coronel-Castillo C, Vera-Barajas A, Contreras-Carmona J, Ponciano-Rodríguez G, Zamora-Valdés D. The cellular pathways of liver fibrosis in non-alcoholic steatohepatitis. Ann Transl Med 2020;8(6):400. doi: 10.21037/atm.2020.02.184 